\begin{tabular}{|l|l|l|c|c|}
\hline Clinical Study & $\begin{array}{l}\text { S S Daskalopoulou, } \\
\text { A B Cooke and others }\end{array}$ & $\begin{array}{l}\text { Irisin and exercise workload in } \\
\text { young subjects }\end{array}$ & $\mathbf{1 7 1 : 3}$ & $\mathbf{3 4 3 - 3 5 2}$ \\
\cline { 1 - 3 }
\end{tabular}

\title{
Plasma irisin levels progressively increase in response to increasing exercise workloads in young, healthy, active subjects
}

\author{
Stella S Daskalopoulou ${ }^{1,2, *,+}$, Alexandra B Cooke ${ }^{1, *}$, Yessica-Haydee Gomez ${ }^{2}$, \\ Andrew F Mutter ${ }^{1}$, Andreas Filippaios ${ }^{4}$, Ertirea T Mesfum ${ }^{3}$ and \\ Christos S Mantzoros ${ }^{3,4}$ \\ ${ }^{1}$ Division of Experimental Medicine, Department of Medicine, Faculty of Medicine, McGill University, \\ Montreal, Quebec, Canada, ${ }^{2}$ Division of Internal Medicine, Department of Medicine, Faculty of Medicine, \\ Research Institute of the McGill University Health Centre, Montreal, Quebec, Canada, ${ }^{3}$ Endocrinology Section, \\ VA Boston Healthcare System, Boston, Massachusetts, USA and ${ }^{4}$ Division of Endocrinology, Diabetes and \\ Metabolism, Harvard Medical School, Beth Israel Deaconess Medical Center, Boston, Massachusetts, USA \\ *(S S Daskalopoulou and A B Cooke contributed equally to this work) \\ ${ }^{+} S$ S Daskalopoulou is now at Montreal General Hospital, 1650 Cedar Avenue, C2.101.4, Montreal, Quebec, \\ Canada H3G 1A4
}

\author{
Correspondence \\ should be addressed \\ to S S Daskalopoulou \\ Email \\ stella.daskalopoulou@ \\ mcgill.ca
}

\begin{abstract}
Background: Irisin, a recently discovered myokine, has been shown to induce browning of white adipose tissue, enhancing energy expenditure and mediating some of the beneficial effects of exercise. We aimed to estimate the time frame of changes in irisin levels after acute exercise and the effect of different exercise workloads and intensities on circulating irisin levels immediately post-exercise.

Methods: In a pilot study, four healthy subjects ( $22.5 \pm 1.7$ years) underwent maximal workload exercise (maximal oxygen consumption, $\mathrm{VO}_{2}$ max ) and blood was drawn at prespecified intervals to define the time frame of pre- and post-exercise irisin changes over a 24-h period. In the main study, 35 healthy, non-smoking (23.0 \pm 3.3 years) men and women $(n=20 / 15)$ underwent three exercise protocols $\geq 48$-h apart, in random order: i) maximal workload $\left(\mathrm{VO}_{2}\right.$ max $)$; ii) relative workload ( $70 \%$ of $\mathrm{VO}_{2} \mathrm{max} / 10 \mathrm{~min}$ ); and iii) absolute workload $(75 \mathrm{~W} / 10 \mathrm{~min}$ ). Blood was drawn immediately pre-exercise and $3 \mathrm{~min}$ post-exercise.

Results: In the pilot study, irisin levels increased by $35 \% 3 \mathrm{~min}$ post-exercise, then dropped and remained relatively constant. In the main study, irisin levels post-exercise were significantly higher than those of pre-exercise after all workloads (all, $P<0.001)$. Post-to-pre-exercise differences in irisin levels were significantly different between workloads $(P=0.001)$, with the greatest increase by $34 \%$ following maximal workload ( $P=0.004$ vs relative and absolute).

Conclusions: Circulating irisin levels were acutely elevated in response to exercise, with a greater increase after maximal workload. These findings suggest that irisin release could be a function of muscle energy demand. Future studies need to determine the underlying mechanisms of irisin release and explore irisin's therapeutic potential.
\end{abstract}

\section{Introduction}

The beneficial effects of regular physical activity have been well established in preventing obesity, diabetes, and its complications, as well as in improving health outcomes (1), but the molecular mechanisms by which these beneficial effects occur are less well understood. Skeletal muscle has recently been shown to play an active role in the regulation of metabolic homeostasis through its ability to communicate with adipose tissue in an (c) 2014 European Society of Endocrinology Printed in Great Britain
Published by Bioscientifica Ltd. 
endocrine manner (2). This cross talk has been reported to mediate many exercise-associated metabolic changes, whereby the contraction of the skeletal muscle causes an enhanced release of several myokines capable of interacting with adipose tissue, such as IL6, IL15, and, most recently, irisin $(2,3,4)$.

Irisin is a recently discovered hormone identified both in mice and humans (4), the levels of which have been reported to increase in response to exercise (4). Irisin has also been proposed to mediate some of the beneficial effects of exercise on metabolism (4). Reportedly regulated by peroxisome proliferator-activated receptor (PPAR)- $\gamma$ co-activator $1 \alpha(P G C 1 \alpha)$, irisin is proteolytically cleaved from the fibronectin type III domain-containing 5 (FNDC5) gene product and secreted into the circulation from skeletal muscle in response to exercise (4). This newly identified myokine is proposed to induce the browning of subcutaneous white adipose tissue and activate thermogenesis by increasing uncoupling protein 1 (UCP1) levels, both in vitro and in vivo (4). In high-fat diet-fed mice, viral injection of full-length Fndc5 resulted in a 15 -fold increase in liver Fndc 5 mRNA levels and a three- to fourfold increase in plasma irisin levels (4), which subsequently led to reduced fasting insulin, improved glucose tolerance, and decreased body weight (4). Together, these results suggest that increased irisin levels can induce the browning of white adipose tissue, increase energy expenditure, and exert both anti-obesity and anti-diabetic effects $(5,6)$. More recently, to confirm the above findings, Zhang et al. (7) have cultured murine adipocytes with recombinant irisin and observed a rapid and significant upregulation of brown cell markers, including $U c p 1$, and $P g_{c} 1 \alpha$. Furthermore, they treated mice fed a high-fat diet with daily injections of recombinant irisin for 14 days and observed similar induction of browning genes, reduction in body weight, and improvements in glucose tolerance (7), as was previously shown using Fndc5 viral vectors (4). These findings highlight irisin's potential to become an attractive therapeutic target to treat metabolic disorders such as obesity and diabetes $(4,7)$.

To date, few human studies have directly looked at the effect of acute exercise on circulating irisin levels $(8,9,10$, $11,12)$. We previously examined the effects of an acute bout of exercise (short sprints) in young healthy men $(n=15)$ and demonstrated that circulating irisin levels were increased $30 \mathrm{~min}$ after completion of acute exercise (8). However, whether irisin increases earlier and/or increases in a dose-dependent manner in response to different workloads and intensities of acute exercise is not known. Furthermore, whether important physiological or demographic variables play a role in prediction of irisin's response to exercise remains to be elucidated. Thus, in our pilot study, we aimed to identify the time frame of irisin release over a period of $24 \mathrm{~h}$. After determining the time point of maximum increase, we aimed in our main study to estimate the effect of acute exercise of different workloads and intensities on circulating irisin levels and explore effect modification by sex, BMI, exercise capacity, and/or baseline physical activity levels.

\section{Subjects and methods}

The study was approved by the ethics and scientific reviews boards of the McGill University Health Centre (MUHC). Written informed consent was obtained from all participants.

\section{Participants}

We recruited consecutive young (aged 18-30 years), healthy, active men and women via notices on Montreal area websites. The International Physical Activity Questionnaire (IPAQ) was used to assess baseline physical activity levels (13); moderate and highly active individuals were recruited. Exclusion criteria were previously diagnosed cardiovascular disease, congenital heart diseases, traditional cardiovascular risk factors (diabetes mellitus, hypertension, and dyslipidemia), metabolic syndrome, renal disease, respiratory diseases, inflammatory/autoimmune diseases, obesity (BMI $\geq 30 \mathrm{~kg} / \mathrm{m}^{2}$ ), pregnancy, and history of ever-smoking. Furthermore, subjects who were acutely ill or on cardioprotective medications (including aspirin) were also excluded.

Upon their first visit, participants completed a questionnaire, including information about past medical history, current medication use, and lifestyle habits. Their height and weight were measured, as well as waist and hip circumferences.

\section{Exercise protocols}

The assessments were performed in a temperature (22 \pm $\left.1{ }^{\circ} \mathrm{C}\right)$ and humidity $(60 \pm 5 \%)$ controlled environment (Vascular Health Unit, MUHC). For the pilot study, participants underwent a maximal workload protocol to volitional exhaustion (maximal oxygen consumption $\left.\left(\mathrm{VO}_{2} \max \right)\right)$ using a treadmill and metabolic cart. The subjects were instructed to perform their regular daily activities but avoid vigorous exercise $24 \mathrm{~h}$ before the exercise session and for the duration of the study post-exercise. 
In the main study II, to induce different levels of physical exercise, each participant performed three different supervised exercise protocols in a random order on three separate study days at least $\geq 48 \mathrm{~h}$ apart from each other. Before each session, participants were asked to abstain from any type of vigorous exercise for $24 \mathrm{~h}$. Participants performed their assessments at the same time of day to reduce any effects of possible circadian variability.

The assessments included: i) maximal workload: the same protocol as the one used in the pilot study, which is a supervised incremental treadmill exercise protocol to volitional exhaustion (modified Bruce protocol, validated in young healthy individuals) (14). A treadmill (Trackmaster, TMX-055) and an automated metabolic cart (Medisoft's Ergocard) to assess $\mathrm{VO}_{2}$ max were used. Time to exercise cessation was recorded. ii) Relative workload: a 10 -min bout of treadmill exercise at $70 \%$ of each subject's $\mathrm{VO}_{2 \text { max }}$, as assessed by the maximal workload session; and iii) absolute workload: a 10-min bout of exercise at $75 \mathrm{~W}$ resistance on a cycle ergometer (Ergoline, Ergoselect 100).

The order of the maximal and absolute workload assessments was randomized using a web-based list randomizer (15). When the maximal workload was randomized to the first assessment, the relative and absolute workloads were further randomized. Brachial blood pressure was measured according to the guidelines (16) using cuff sphygmomanometry (HEM-705CP, Omron Corp. St. Charles, IL, USA) pre-exercise (baseline) and 2 min post-exercise.

\section{Determination of peak metabolic equivalents}

$\mathrm{VO}_{2}$ max values were calculated as $\mathrm{ml} /\left(\mathrm{kg} / \mathrm{m}^{2}\right)$ from the breath-by-breath analysis provided by the automated metabolic cart. Peak metabolic equivalents (METs) were then determined by dividing the calculated $\mathrm{VO}_{2}$ peak by the resting metabolic rate (one MET) of each individual participant, as calculated by the Harris-Benedict equation (17).

\section{Blood and urine collection}

In the pilot study, participants had their blood drawn (4 ml) by a research nurse using a peripheral venous catheter (Becton, Dickinson, and Company, Franklin Lakes, NJ, USA) immediately pre-exercise, $3 \mathrm{~min}$ post-exercise, as well as $10 \mathrm{~min}, 30 \mathrm{~min} 1,2,3,6,12$, and $24 \mathrm{~h}$ post-exercise. The catheter set was introduced and well secured on the forearm 30 min pre-exercise and removed at the end of the session. In all four subjects, urine samples were also obtained preexercise in sterilized urine cups (Starplex Scientific,
Cleveland, OH, USA); $15 \mathrm{ml}$ was collected into $15 \mathrm{ml}$ conical tubes (Thermo Fisher Scientific, Hampton, NH, USA). After centrifugation, plasma and urine were processed and stored at $-80^{\circ} \mathrm{C}$ for the analysis of irisin (plasma and urine) and lactate (plasma only).

In the main study, the time of the post-exercise blood collection (3 $\mathrm{min}$ ) was selected as the most appropriate based on the results of the pilot study demonstrating the largest increase in irisin levels $3 \mathrm{~min}$ post-exercise. Therefore, in the main study, blood was collected from all participants immediately pre-exercise $(10 \mathrm{ml})$ and $3 \mathrm{~min}$ post-exercise $(10 \mathrm{ml})$ through a peripheral venous catheter. The blood samples were collected, processed, and stored in the same manner as indicated earlier.

Plasma irisin levels were quantified using irisin ELISA (Aviscera Bioscience (Santa Clara, CA, USA), now provided by Phoenix Pharmaceuticals (Burlingame, CA, USA) under the same cat\# EK-067-52) (8). Before using this ELISA kit with clinical samples, we performed a number of validation studies. The detectable range of the kit was $0.066-1024 \mathrm{ng} / \mathrm{ml}$, with intra-assay variation and interassay variation being $0.6-4.8$ and $8.0-10.0 \%$ respectively. Our results showed that serum and plasma concentrations of irisin were relatively similar, and that multiple freezethaw cycles $(0,2,4,8)$ did not cause any significant changes in irisin levels. The assessment of linearity in serum and plasma samples revealed a $124 \%$ recovery for the $1 / 2$ dilution, and higher recovery (up to $213 \%$ ) in the $1 / 4$ and $1 / 8$ dilutions. To the best of our knowledge, no commercially available assay to date can differentiate between irisin and soluble FNDC5 as there is $100 \%$ crossreactivity between the two proteins. However, the EK-06752 kit by Phoenix Pharmaceuticals is the most widely used and validated kit for the assessment of plasma irisin in the current literature.

Plasma lactate was measured using automated Roche cobas c311 analyzer (Roche Diagnostics) via enzymatic colorimetric method.

All analyses were performed in a blinded manner under code by study personnel unaware of the hypotheses of the study.

\section{Statistical analyses}

Descriptive statistics were used to present baseline characteristics. The distribution of the data was tested and parametric or non-parametric tests were used accordingly. Differences between pre- and post-exercise irisin levels were compared for each session using Wilcoxon signed-rank test. The post-to-pre-exercise change in 


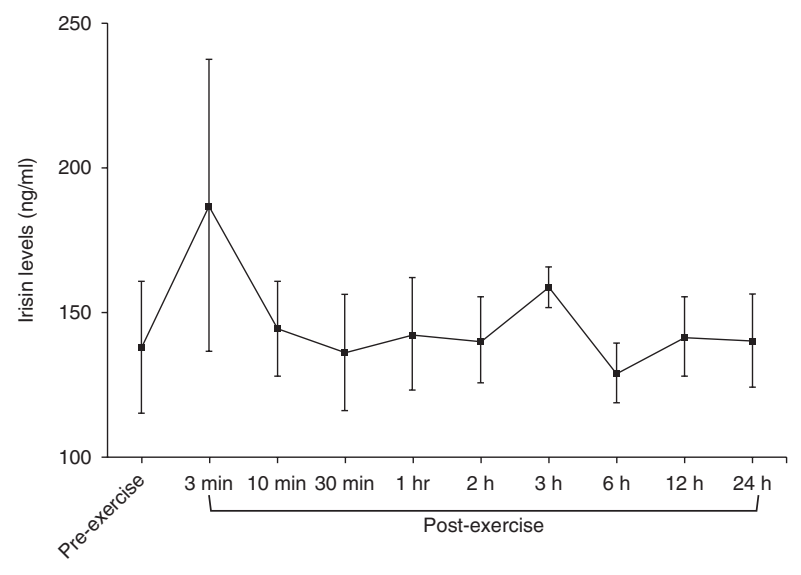

\section{Figure 1}

Irisin levels pre-exercise and post-exercise, and 24-h. Irisin levels were measured pre-exercise and post-exercise at 3-, 10-, 30-min and at 1-, 2-, 3-, 6-, 12-, and 24-h ( $n=4)$. At each time point irisin values are represented as mean \pm s.E.M.

circulating irisin levels was calculated and expressed as the difference between post- and pre-exercise irisin levels. Irisin levels (pre-exercise levels, post-exercise levels, and post-pre difference) were compared among the different exercise intensities using Friedman's test. Kruskal-Wallis test was conducted for comparisons between irisin levels across $\mathrm{VO}_{2}$ max tertiles. We further performed Pearson's or Spearman's rank correlations between irisin after maximal workload (pre-exercise levels, post-exercise levels, and post-pre difference, separately) and BMI, IPAQ, lactate levels, $\mathrm{VO}_{2}$ max, and other exercise parameters. Analysis of covariance (ANCOVA) was used to assess between-group differences for sex, BMI, IPAQ, and $\mathrm{VO}_{2}$ max in irisin levels on all three workloads. We used repeated measures ANCOVA and multivariate ANCOVA (MANCOVA) to test interactions between certain variables (sex, BMI, and, IPAQ or $\mathrm{VO}_{2 \text { max }}$ ) and irisin levels on the three workloads (adjustment included age, sex, BMI, and $\mathrm{VO}_{2}$ max accordingly). IBM SPSS Statistics version 20.0 (Armonk, NY, USA) was used.

\section{Results}

\section{Pilot study}

We recruited four young (mean age \pm s.D.: $22.5 \pm 1.7$ ), healthy, non-smoking individuals (two men and two women). Irisin levels increased by $35 \%(137.9 \pm 45.7$ to
$187.3 \pm 101.0 \mathrm{ng} / \mathrm{ml}) 3 \mathrm{~min}$ post-exercise, compared with the baseline value. At time points later than $3 \mathrm{~min}$, levels dropped toward baseline and remained relatively constant throughout the following time frame of $24 \mathrm{~h}$ (Fig. 1). Based on these findings, which showed that the greatest increase in irisin was reported $3 \mathrm{~min}$ post-exercise, this time point was selected for blood collection in the main study. Moreover, we report for the first time the presence of irisin in the urine at a mean concentration of $14.47 \pm$ $4.46 \mathrm{ng} / \mathrm{ml}$ pre-exercise.

\section{Main study}

We recruited 35 subjects (mean age \pm s.D.: $23 \pm 3.3$ years; 20 men and 15 women); baseline characteristics are presented in Table 1 . On the maximal workload session, all subjects achieved a respiratory exchange rate (ratio of the volume of carbon dioxide produced and the volume of oxygen used, i.e. $\mathrm{VCO}_{2} / \mathrm{VO}_{2}$ ) of $>1.1$ (mean \pm s.D.: 1.2 \pm 0.1 ); this indicates adequate exercise effort (secondary endpoint criterion of reaching $\mathrm{VO}_{2} \max$ ) (18). The average exercise time on the maximal workload session was 18.0 $\pm 3.1 \mathrm{~min}$.

The mean pre-exercise irisin level and post-exercise irisin level for the three exercise workloads are presented in Table 1 . There were no significant differences in pre-exercise irisin levels on the three exercise workload sessions $(P=0.581)$. The irisin post-pre difference was significant in response to all three workloads $(P<0.001)$ (Fig. 2$)$.

Table 1 Subject baseline characteristics. The independent samples Student's $t$-test was used to compare baseline characteristics between men and women.

\begin{tabular}{|c|c|c|c|c|}
\hline & $\begin{array}{l}\text { Whole } \\
\text { cohort }\end{array}$ & Men & Women & $\begin{array}{c}P \\
\text { value }\end{array}$ \\
\hline $\begin{array}{l}\text { Sex (men/ } \\
\text { women) }\end{array}$ & $20 / 15$ & 20 & 15 & - \\
\hline Age (years) & $23.0 \pm 3.3$ & $24.2 \pm 3.9$ & $21.4 \pm 1.4$ & 0.007 \\
\hline Height $(\mathrm{cm})$ & $173.8 \pm 9.5$ & $180.3 \pm 6.3$ & $165.2 \pm 4.9$ & 0.000 \\
\hline Weight (kg) & $68.01 \pm 11.8$ & $75.6 \pm 9.1$ & $57.9 \pm 5.8$ & 0.000 \\
\hline BMI $\left(\mathrm{kg} / \mathrm{m}^{2}\right)$ & $22.4 \pm 2.5$ & $23.2 \pm 2.5$ & $21.2 \pm 1.9$ & 0.015 \\
\hline Waist $(\mathrm{cm})$ & $78.1 \pm 10.3$ & $83.5 \pm 8.0$ & $71.1 \pm 8.6$ & 0.000 \\
\hline Hip (cm) & $89.1 \pm 9.2$ & $91.2 \pm 8.6$ & $86.6 \pm 9.7$ & 0.164 \\
\hline $\begin{array}{l}\text { Waist:hip } \\
\text { ratio }\end{array}$ & $0.9 \pm 0.1$ & $0.9 \pm 0.1$ & $0.8 \pm 0.1$ & 0.008 \\
\hline $\mathrm{IPAQ}^{\mathrm{a}}$ & $\begin{array}{c}2919(1831- \\
4548)\end{array}$ & $\begin{array}{c}2750(1428- \\
6152)\end{array}$ & $\begin{array}{c}2919(2239- \\
3945)\end{array}$ & 0.894 \\
\hline
\end{tabular}

IPAQ, International Physical Activity Questionnaire.

${ }^{\text {aP }}$ Presented as median (interquartile range), all other values are mean + s.D. 


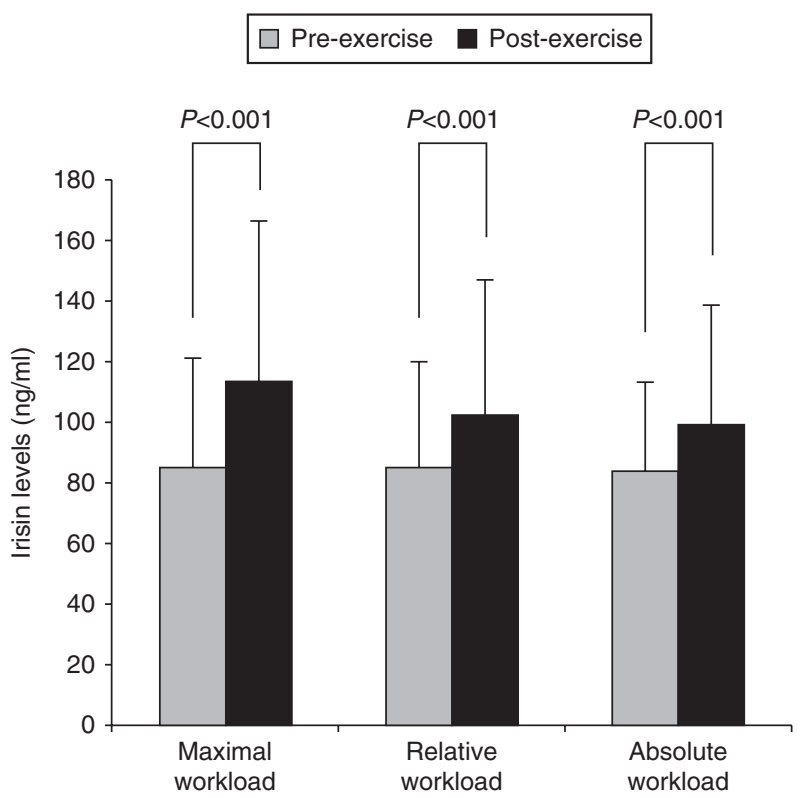

\section{Figure 2}

Irisin levels pre-exercise and post-exercise on the three workload sessions. Post-exercise irisin levels are significantly higher than pre-exercise irisin levels on all three workload sessions $(n=35)$. Data were shown as mean +s.D. $P$ values were obtained using Wilcoxon signed-rank test to compare post- and pre-exercise irisin levels.

\section{Different exercise workloads in relation to post-pre-exercise difference in irisin levels}

A dose-response increase in irisin levels was noted according to the exercise workload $(P=0.001)$, with the greatest and lowest increase observed after the maximal and absolute workload respectively (Table 2 ). The post-pre differences in irisin levels between maximum and relative workloads, and between maximum and absolute workloads, were all significant (both, $P=0.004$ ). The post-pre difference in irisin levels, however, was not significantly different between the relative and absolute workloads.

\section{Different exercise workloads in relation to post-pre-exercise difference in lactate levels}

Baseline lactate pre-exercise levels were not significantly different among the three exercise workload sessions. However, the post-pre increment in lactate levels was significantly different across workloads $(P<0.001)$, with the greatest increase after the maximal workload protocol (Table 2).

\section{Correlation between incremental changes of irisin and lactate levels}

The lactate post-pre incremental change was correlated with the corresponding change in irisin levels post-pre on each workload session (Table 3), with the strongest correlation observed during the maximal workload $\left(r_{\mathrm{s}}=0.523, P=0.001\right)$.

Significant positive correlations were also observed between lactate post-pre-exercise after maximal workload and $\mathrm{VO}_{2 \text { max }}\left(r_{\mathrm{s}}=0.471, P=0.004\right)$, exercise time $\left(r_{\mathrm{s}}=0.528\right.$, $P=0.001)$, and peak METs $\left(r_{\mathrm{s}}=0.477, P=0.004\right)$.

Interactions between workloads and other parameters in predicting changes in irisin levels

No significant interactions were found between the different exercise workloads and sex, BMI, IPAQ, and $\mathrm{VO}_{2}$ max on changes in irisin levels (pre-exercise, postexercise, and post-pre difference) $(P>0.05)$.

\section{$\mathrm{VO}_{2}$ max and other exercise parameters}

After maximal workload, $\mathrm{VO}_{2}$ max was positively correlated with irisin levels post-exercise $\left(r_{p}=0.393, P=0.020\right)$ as well as irisin post-pre difference $\left(r_{\mathrm{s}}=0.414, P=0.013\right)$. Furthermore, when dividing the study sample into tertiles based on their $\mathrm{VO}_{2}$ max, we found a trend toward a progressive increase in irisin post-pre difference across the $\mathrm{VO}_{2} \max$ groups $(P=0.059)$ (Table 4$)$ on the maximal workload session. Similarly, $\mathrm{VO}_{2 \text { max }}$ was also positively correlated with lactate post-pre difference $\left(r_{\mathrm{s}}=0.471, P=0.004\right)$ after maximal workload. Lactate levels displayed a progressive increase across the $\mathrm{VO}_{2}$ max groups $(P=0.017)$ (Table 4$)$.

Table 2 Irisin and lactate levels on all three workload sessions. Irisin levels $(\mathrm{ng} / \mathrm{ml})$, lactate levels $(\mathrm{mmol} / \mathrm{l})$. All values are mean \pm s.D. The Friedman's test was used to compare irisin and lactate values between all three workload sessions.

\begin{tabular}{|c|c|c|c|c|}
\hline & $\begin{array}{l}\text { Maximal } \\
\text { workload }\end{array}$ & $\begin{array}{l}\text { Relative } \\
\text { workload }\end{array}$ & $\begin{array}{l}\text { Absolute } \\
\text { workload }\end{array}$ & $P$ value \\
\hline \multicolumn{5}{|l|}{ Irisin } \\
\hline Pre-exercise & $84.8 \pm 36.1$ & $85.1 \pm 35.0$ & $83.4 \pm 29.6$ & 0.581 \\
\hline Post-exercise & $113.3 \pm 52.7$ & $102 \pm 44.2$ & $99.1 \pm 39.3$ & 0.008 \\
\hline $\begin{array}{l}\text { Post-pre } \\
\text { difference }\end{array}$ & $28.5 \pm 39.3$ & $17.4 \pm 14.3$ & $15.7 \pm 17.8$ & 0.001 \\
\hline \multicolumn{5}{|l|}{ Lactate } \\
\hline Pre-exercise & $2.1 \pm 2.4$ & $1.6 \pm 0.6$ & $1.5 \pm 0.4$ & 0.155 \\
\hline Post-exercise & $12.3 \pm 4.5$ & $3.6 \pm 2.1$ & $2.5 \pm 1.8$ & $<0.001$ \\
\hline $\begin{array}{l}\text { Post-pre } \\
\text { difference }\end{array}$ & $10.2 \pm 6.3$ & $2.0 \pm 2.1$ & $1.0 \pm 1.9$ & $<0.001$ \\
\hline
\end{tabular}


Table 3 Correlations between lactate and irisin levels on all three workload sessions. Irisin levels ( $\mathrm{ng} / \mathrm{ml})$, lactate levels $(\mathrm{mmol} / \mathrm{l})$. A Spearman's correlations coefficient was used. Significant correlations are in bold.

\begin{tabular}{|c|c|c|}
\hline & Lactate post-exercise & $\begin{array}{l}\text { Lactate post- } \\
\text { pre difference }\end{array}$ \\
\hline \multirow{3}{*}{$\begin{array}{l}\text { Maximal workload } \\
\text { Irisin post-exercise }\end{array}$} & & \\
\hline & $r=0.487$ & \\
\hline & $P=0.003$ & \\
\hline \multirow{2}{*}{$\begin{array}{l}\text { Irisin post-pre } \\
\text { difference }\end{array}$} & $r=0.838$ & $r=0.523$ \\
\hline & $P=0.000$ & $P=0.001$ \\
\hline \multicolumn{3}{|l|}{ Relative workload } \\
\hline Irisin post-exercise & $\begin{array}{l}r=0.415 \\
P=0.007\end{array}$ & \\
\hline \multirow{5}{*}{$\begin{array}{l}\text { Irisin post-pre } \\
\text { difference } \\
\text { Absolute workload } \\
\text { Irisin post-exercise }\end{array}$} & $r=0.604$ & $r=0.372$ \\
\hline & $P=0.000$ & $P=0.014$ \\
\hline & & \\
\hline & $r=-0.263$ & \\
\hline & $P=0.064$ & \\
\hline \multirow{2}{*}{$\begin{array}{l}\text { Irisin post-pre } \\
\text { difference }\end{array}$} & $r=0.508$ & $r=-0.229$ \\
\hline & $P=0.000$ & $P=0.093$ \\
\hline
\end{tabular}

Exercise time and peak METs were significantly different between $\mathrm{VO}_{2}$ max tertiles, with the highest values observed in subjects who reached a higher $\mathrm{VO}_{2 \text { max }}$. Furthermore, irisin post-pre difference after maximal exercise was correlated with peak METs $\left(r_{\mathrm{s}}=0.451\right.$, $P=0.007)$ and trended toward significance with exercise time $\left(r_{\mathrm{s}}=0.301, P=0.079\right)$, while post-exercise irisin was significantly correlated with exercise time $\left(r_{p}=0.357, P=0.035\right)$.

Energy expenditure (kcal) was significantly different between the three different workload sessions (maximal: $180.27 \pm 80.90 \mathrm{kcal}$; relative: $111.90 \pm 34.32 \mathrm{kcal}$; absolute: $62.08 \pm 18.29 \mathrm{kcal}) \quad(P<0.001)$. Furthermore, energy expenditure between any two workloads (maximal vs relative, maximal vs absolute, and relative vs absolute) was significant (all $P<0.001$ ). On the maximal workload session, there is a trend for a positive correlation between energy expenditure and irisin post-exercise levels $\left(r_{\mathrm{s}}=0.321, P=0.064\right)$ and irisin post-pre difference $\left(r_{\mathrm{s}}=0.307, P=0.078\right)$. Furthermore, energy expenditure was significantly correlated with lactate post-pre difference $\left(r_{\mathrm{s}}=0.594, P<0.001\right)$.

\section{Discussion}

We report for the first time the time frame of irisin changes in plasma and urine, as well as a significant and progressive increase in the levels of plasma irisin with increasing exercise workload immediately post-exercise. More specifically, we showed that irisin is significantly elevated early, with the highest irisin levels increased by $35 \%$, occurring $3 \mathrm{~min}$ post-exercise in our pilot study, and by $34 \%$ in our main study of 35 subjects. Irisin is a myokine released in response to exercise in rodents, but few studies to date have looked at the effect of acute exercise in humans. We demonstrated in a previous acute exercise study in humans that circulating irisin levels increase $30 \mathrm{~min}$ after short sprints in young healthy moderately trained men (8). Norheim et al. (10) also performed an acute exercise test (45 min of ergometer cycling at $\left.70 \% \mathrm{VO}_{2} \max \right)$ in young healthy subjects $(n=13)$ and showed significantly higher levels (1.2-fold) immediately after exercise, which returned to pre-exercise levels at $2 \mathrm{~h}$ post-exercise. They did not observe any effect of acute exercise on FNDC5 mRNA; however, PGC1 $\alpha$ was 7.4-fold higher $2 \mathrm{~h}$ post-exercise when compared with pre-exercise

Table 4 Exercise parameters, irisin, and lactate levels in the $\mathrm{VO}_{2}$ max groups (low, moderate, and high) on the maximal workload sessions. All values are mean \pm s.D. The Kruskal-Wallis test was used to compare exercise parameters, irisin values, and lactate levels between $\mathrm{VO}_{2}$ max groups.

\begin{tabular}{l} 
\\
\hline $\mathrm{VO}_{2}$ max \\
Exercise time \\
Peak METs \\
Energy expenditure \\
Irisin pre-exercise \\
Irisin post-exercise \\
Irisin post-pre difference \\
Lactate pre-exercise \\
Lactate post-exercise \\
Lactate post-pre difference
\end{tabular}

\begin{tabular}{c}
\hline Low $\mathbf{V O}_{2 \max }(n=12)$ \\
\hline $38.5 \pm 4.1$ \\
$15.3 \pm 1.9$ \\
$11.3 \pm 1.5$ \\
$84.2 \pm 17.3$ \\
$74.3 \pm 18.7$ \\
$91.6 \pm 27.9$ \\
$17.3 \pm 20.3$ \\
$2.4 \pm 3.2$ \\
$10.1 \pm 4.3$ \\
$7.7 \pm 6.9$
\end{tabular}

\begin{tabular}{c} 
Moderate $\mathbf{V O}_{2 \max }(n=12)$ \\
\hline $48.5 \pm 3.0$ \\
$18.0 \pm 1.3$ \\
$14.4 \pm 1.3$ \\
$133.8 \pm 45.7$ \\
$83.4 \pm 16.0$ \\
$113.2 \pm 51.8$ \\
$29.8 \pm 42.6$ \\
$2.5 \pm 2.6$ \\
$12.0 \pm 5.4$ \\
$9.5 \pm 7.4$
\end{tabular}

\begin{tabular}{c} 
High $\mathbf{V O}_{2 \max }(n=11)$ \\
\hline $60.6 \pm 5.6$ \\
$21.1 \pm 2.6$ \\
$17.6 \pm 1.6$ \\
$227.1 \pm 52.1$ \\
$97.8 \pm 58.6$ \\
$137.1 \pm 66.8$ \\
$39.3 \pm 19.7$ \\
$1.3 \pm 0.2$ \\
$14.9 \pm 2.1$ \\
$13.6 \pm 2.1$
\end{tabular}

\begin{tabular}{r}
\hline P value \\
\hline$<0.001$ \\
$<0.001$ \\
$<0.001$ \\
$<0.001$ \\
0.345 \\
0.101 \\
0.059 \\
0.081 \\
0.018 \\
0.017
\end{tabular}

Energy expenditure (kcal); exercise time ( $\mathrm{min})$; irisin levels $(\mathrm{ng} / \mathrm{ml})$; lactate levels (mmol/l); peak METs, peak metabolic equivalent; $\mathrm{VO}{ }_{2}$ max, maximal oxygen consumption ( $\mathrm{ml} / \mathrm{kg}$ per $\mathrm{min}$ ). 
values (10). In a study by Kraemer et al. (9) young subjects $(n=12)$ completed $90 \mathrm{~min}$ of treadmill exercise at $60 \%$ of $\mathrm{VO}_{2}$ max and irisin levels were measured during exercise (at 0,54 , and $90 \mathrm{~min}$ ) as well as at $20 \mathrm{~min}$ post-exercise. They observed significantly higher irisin levels at $54 \mathrm{~min}$ into exercise test $(20.4 \%$ in young males and $22.5 \%$ in young women). However, irisin levels were no longer elevated at $90 \mathrm{~min}$ post-exercise, as well as $20 \mathrm{~min}$ postexercise. These results contribute new evidence to regulation of irisin levels during steady-state acute physical activity, suggesting that irisin signaling occurs during the first hour of exercise (9). To date, the majority of studies have measured irisin levels in plasma or serum; however, Aydin et al. (12) provide the first demonstration of irisin in the saliva, supported by their results showing strong irisin immunoreactivity in human submandibular glands. Interestingly, they observed a significant increase in irisin levels in the saliva after moderate outdoor exercise (5.5 km/45 min) in a middle-aged normal weight population $(n=14)(12)$. There remain two studies $(11,12)$ which have shown no significant changes in plasma irisin in response to acute exercise. However, the timing of blood draw post-exercise was either not exactly specified (12) or $3 \mathrm{~h}$ after the exercise test (11). In addition to the findings from Kraemer et al. (9), the results from our pilot study lend support to an acute and transient increase in plasma irisin, which may explain some of the inconsistent results when irisin was measured at later time points post-exercise.

We showed for the first time that the increase in irisin levels after maximal workload protocol was significantly greater in more fit individuals, who exercised longer and reached a higher percentage of $\mathrm{VO}_{2}$ max and peak METs post-exercise providing the dose-response criterion for causality as per Bradford Hill criteria (19). These subjects also had a greater increase in lactate in response to maximal exercise, suggestive of the greater muscle strain they underwent. The change in irisin and lactate levels correlated, and both increased with higher exercise workload, suggesting that greater increase in irisin levels in these individuals could perhaps be a function of muscle energy demands. Furthermore, our hypothesis that muscle demand may play a role in the physiological regulation of irisin concurs with the findings reported by Huh et al. (8), who showed that the increase in irisin after acute exercise correlated with a decrease in muscle ATP levels. This suggests that lower ATP levels and/or higher ADP+ phosphate group (Pi) may act to signal irisin release to help restore ATP homeostasis in the working muscle (8). We did not observe significant differences in baseline irisin levels amongst individuals with different levels of physical fitness, but this may be due to the inclusion of only physically active individuals, as defined by IPAQ scores. We do, however, report a greater increase in irisin levels in response to higher workload exercise, with the greatest increase observed in more physically fit individuals who were able to reach a higher $\mathrm{VO}_{2}$ max within the maximal workload session.

We have demonstrated that short-term, highintensity exercise leads to the most significant increase in plasma irisin levels. These findings lend support to the positive effects of high-intensity exercise as an effective and time-efficient method of improving cardiorespiratory fitness and cardiac mass in sedentary adults (20). However, we have also demonstrated for the first time that even a total duration of $10 \mathrm{~min}$ of sub-maximal exercise (relative and absolute workloads) increase circulating irisin levels. This is especially interesting since maximal exercise to exhaustion is not a common daily occurrence for most people. In fact, several studies have suggested that shorter bouts of exercise accumulated through several episodes $(<10 \mathrm{~min})$ are as beneficial as longer bouts of activity (>30 min) (21). Recent health guidelines have stated that the accumulation of at least $150 \mathrm{~min}$ of aerobic physical activity, even if done in short bouts of $10 \mathrm{~min}$, has beneficial health outcomes, namely effective weight loss and decreased risk for diabetes (22). It remains to be evaluated whether these beneficial effects are at least partly attributed to smaller and more frequent irisin release after several short bouts of low workload exercise.

We noted significant elevated irisin levels postexercise on all three workloads. Physiologically, it remains unclear whether this rapid increase in circulating irisin levels results from a de novo production of FNDC5 in response to exercise, or whether FNDC5 is pre-translated, stored, and only released into the circulation as muscle demand increases. Alternatively, exercise may rapidly activate a cleavage factor specific to FNDC5, leading to the rapid release of irisin into the circulation following exercise initiation. We can hypothesize from a study reported by Norheim et al. (10) that the acute increase in plasma irisin levels is probably independent of an increase in the de novo production of FNDC5, as the muscle FNDC5 mRNA levels were unchanged after the acute exercise bout. Further studies, however, will be needed to confirm the mechanisms behind the very rapid release of irisin into the bloodstream.

To date, there is no evidence in the literature regarding irisin metabolism, and whether the kidney may play a role in its excretion. We have for the first 
time successfully measured irisin levels at physiological concentrations in the urine, which suggests a role for the kidney in the metabolism and excretion of circulating irisin. This topic will be part of our future research.

We reported no significant interactions between different exercise workloads and BMI, IPAQ, and $\mathrm{VO}_{2}$ max on irisin levels, demonstrating that elevated irisin levels after exercise is driven by the differences in exercise workload alone. BMI was previously shown to be the main contributor to circulating irisin levels at rest $(8,23,24)$. In a wide range of BMI $\left(8.9-84.6 \mathrm{~kg} / \mathrm{m}^{2}\right)$, significant correlations of irisin with body weight, BMI, fat mass, and muscle mass were noted (23). Huh et al. (8) report a trend toward a positive correlation between irisin and BMI in healthy middle-aged women, as well as a decrease in irisin levels in obese individuals 6 months postbariatric surgery. However, a negative correlation was found in a study of 29 morbidly obese men (24). We did not observe any relationship between BMI and irisin levels at baseline or after exercise; however, individuals in our study had a narrow and healthy range of BMI $\left(22.4 \pm 2.5 \mathrm{~kg} / \mathrm{m}^{2}\right)$. Interestingly, men had significantly higher BMI than women. Although non-significant, possibly due to small sample size, they also had higher baseline irisin levels and a greater increase post-exercise. This could perhaps be explained by increased muscle mass in men, because Huh et al. (8) showed that circulating irisin levels were positively correlated with bicep circumference, a surrogate marker of muscle mass. This suggests that increased muscle mass may be the main predictor of irisin levels; however, larger studies will be necessary to draw more definite conclusions.

Limitations of this study include a relatively small and arbitrary sample size, because no prior studies were available on which to base calculations. However, the sample size is larger than that in most previous studies and the several measurement time points increased the power of the study. These data could be used for calculating sample size in future studies. Based on our results, to have adequate power to determine sex differences in irisin levels in response to exercise we would need 62 subjects per group; however, a much larger sample size would be necessary to show significant differences for BMI, IPAQ, and $\mathrm{VO}_{2}$ max. As a result of strict inclusion criteria, we were only able to assess a very narrow and healthy range of BMI, and overall, a very active group of young individuals. Although it was beyond the scope of this study, assessing the above-mentioned associations is an interesting future direction. We did not directly measure muscle mass, and we can only speculate that higher BMI in our group represents higher muscle mass in these young, healthy, active subjects. We did not measure ATP levels; however, we calculated energy expenditure using $\mathrm{VO}_{2}$ and respiratory exchange ratio (RER) values to indirectly assess muscle energy expenditure and ATP utilization (25). Furthermore, we used a cycle ergometer to produce the exercise protocol that corresponded on average to $41.32 \pm 18.8 \% \mathrm{VO}_{2}$ max of every subject (obtained by calculating each subject's average $\mathrm{VO}_{2} / \mathrm{min}$ for each minute of exercise divided by their $\mathrm{VO}_{2}$ max on the maximal workload session). We used this design by convention, so as to study not only a low workload protocol but also evaluate the effect of absolute workload on irisin levels. The cycle ergometer allowed for greater control in setting the same absolute workload for all subjects, as it enables adjustment to each individual's pedal speed to provide the necessary resistance to maintain a $75 \mathrm{~W}$ output. Lastly, we did not obtain hematocrit and hemoglobin values and were not able to adjust for delta plasma volume in our study preexercise and post-exercise. To date, only one other irisin study has examined plasma volume shift after acute exercise and found only a $-3.0 \pm 6.09 \%$ shift in plasma volume after $54 \mathrm{~min}$ at treadmill exercise at $60 \% \mathrm{VO}_{2} \max$ in young healthy subjects (9). In our study, the mean exercise time on the maximal workload session was 18:04 min, and the two other sessions were only $10 \mathrm{~min}$, therefore the minimal shift that might have occurred during our study is unlikely to increase plasma irisin to the magnitude that we have reported.

Strengths of the study include the blinded assessment of irisin levels by personnel who were not aware of the study hypothesis. Furthermore, our pilot study allowed us to identify the optimal time frame that circulating irisin should be measured post-exercise ( $3 \mathrm{~min}$ ). Also, as described in the Methods section in detail, we carried out our own validation studies on the Aviscera/Phoenix Pharmaceutical kit to ensure it would be able to reliably and accurately assess plasma irisin levels in our samples. This is currently the most widely used and validated kit for measuring plasma irisin $(8,10,11,12,24,26,27,28,29)$. These validation studies help to clarify some of the controversies in the literature regarding the validity of the various assays used for measurements of plasma irisin. For instance, Erickson (30) and Raschke et al. (31) have challenged the existence of irisin in humans, and have questioned the initial results of Bostrom et al. on the basis of poor assay validity. However, Bostrom et al. (32) have addressed many of these controversies in a recent editorial with supporting evidence from the literature. Western blot analysis using the Aviscera/Phoenix antibody detects 
a $25 \mathrm{kDa}$ band in plasma, which represents the fully glycosylated form of irisin as demonstrated by Zhang et al. and Moreno-Navarrete et al. (7, 24). Bostrom et al. (32) have shown that the same antibody detects spiked irisin in the plasma at physiological concentrations. Furthermore, in our study, we demonstrated a clear dose-response relationship between exercise intensities and circulating irisin levels, which would be undetectable in the case of an unspecific assay.

In summary, we demonstrated that acute exercise significantly increases circulating irisin levels, and for the first time a significantly greater increase in irisin levels with increasing exercise workload to exhaustion was reported immediately post-exercise. Furthermore, elevated plasma irisin levels after maximal exercise may be a function of muscle energy workload, as suggested by our finding that more physically fit subjects reaching a higher percentage of $\mathrm{VO}_{2}$ max had a greater increase in irisin and lactate levels. The fact that irisin levels were also significantly higher after only a 10-min low-workload exercise might be related to the current clinical evidence that accumulated 10-min exercise bouts could still confer health benefits.

Our important results highlight the need for a more comprehensive understanding of the metabolic regulation of irisin at baseline and the full time frame of the changes in plasma irisin levels after exercise. Furthermore, the relative contributions of different increases in circulating irisin related to repeated exercise sessions of different workloads in improving metabolic disease profiles needs to be investigated.

\section{Declaration of interest}

The authors declare that there is no conflict of interest that could be perceived as prejudicing the impartiality of the research reported.

\section{Funding}

This study was funded by a grant from the Canadian Institutes of Health Research (MOP\#102626) to S S Daskalopoulou.

\section{Author contribution statement}

S S Daskalopoulou obtained funding for the study. S S Daskalopoulou, A B Cooke, Y-H Gomez, and A F Mutter were involved in study concept and design. S S Daskalopoulou, A B Cooke, Y-H Gomez, A F Mutter, A Filippaios, E T Mesfum, and C S Mantzoros were involved in data acquisition, analysis, and interpretation. S S Daskalopoulou, A B Cooke, and Y-H Gomez drafted the manuscript and S S Daskalopoulou, A B Cooke, Y-H Gomez, A F Mutter, A Filippaios, E T Mesfum, and C S Mantzoros were involved in critically revising the manuscript. All authors have read and approved the final version of the manuscript.

\section{References}

1 Shrestha P \& Ghimire L. A review about the effect of life style modification on diabetes and quality of life. Global Journal of Health Science 20124 185-190. (doi:10.5539/gjhs.v4n6p185)

2 Pedersen BK \& Febbraio MA. Muscles, exercise and obesity: skeletal muscle as a secretory organ. Nature Reviews. Endocrinology 20128 457-465. (doi:10.1038/nrendo.2012.49)

3 Pedersen BK, Akerstrom TC, Nielsen AR \& Fischer CP. Role of myokines in exercise and metabolism. Journal of Applied Physiology 2007103 1093-1098. (doi:10.1152/japplphysiol.00080.2007)

4 Bostrom P, Wu J, Jedrychowski MP, Korde A, Ye L, Lo JC, Rasbach KA, Bostrom EA, Choi JH, Long JZ et al. A PGC1- $\alpha$-dependent myokine that drives brown-fat-like development of white fat and thermogenesis. Nature 2012481 463-468. (doi:10.1038/nature10777)

5 Swick AG, Orena S \& O'Connor A. Irisin levels correlate with energy expenditure in a subgroup of humans with energy expenditure greater than predicted by fat free mass. Metabolism 201362 1070-1073. (doi:10.1016/j.metabol.2013.02.012)

6 Polyzos SA, Kountouras J, Shields K \& Mantzoros CS. Irisin: a renaissance in metabolism? Metabolism 201362 1037-1044. (doi:10.1016/j.metabol.2013.04.008)

7 Zhang Y, Li R, Meng Y, Li S, Donelan W, Zhao Y, Qi L, Zhang M, Wang $\mathrm{X}$, Cui $\mathrm{T}$ et al. Irisin stimulates browning of white adipocytes through mitogen-activated protein kinase p38 MAP kinase and ERK MAP kinase signaling. Diabetes 201463 514-525. (doi:10.2337/ db13-1106)

8 Huh JY, Panagiotou G, Mougios V, Brinkoetter M, Vamvini MT, Schneider BE \& Mantzoros CS. FNDC5 and irisin in humans: I. Predictors of circulating concentrations in serum and plasma and II. mRNA expression and circulating concentrations in response to weight loss and exercise. Metabolism 201261 1725-1738. (doi:10.1016/j. metabol.2012.09.002)

9 Kraemer RR, Shockett P, Webb ND, Shah U \& Castracane VD. A transient elevated irisin blood concentration in response to prolonged, moderate aerobic exercise in young men and women. Hormone and Metabolic Research 201446 150-154. (doi:10.1055/s-0033-1355381)

10 Norheim F, Langleite TM, Hjorth M, Holen T, Kielland A, Stadheim HK Gulseth HL, Birkeland KI, Jensen J \& Drevon CA. The effects of acute and chronic exercise on PGC- $1 \alpha$, irisin and browning of subcutaneous adipose tissue in humans. FEBS Journal 2014281 739-749. (doi:10.1111/febs.12619)

11 Pekkala S, Wiklund PK, Hulmi JJ, Ahtiainen JP, Horttanainen M, Pollanen E, Makela KA, Kainulainen H, Hakkinen K, Nyman K et al. Are skeletal muscle FNDC5 gene expression and irisin release regulated by exercise and related to health? Journal of Physiology 2013591 5393-5400. (doi:10.1113/jphysiol.2013.263707)

12 Aydin S, Aydin S, Kuloglu T, Yilmaz M, Kalayci M, Sahin I \& Cicek D. Alterations of irisin concentrations in saliva and serum of obese and normal-weight subjects, before and after $45 \mathrm{~min}$ of a Turkish bath or running. Peptides 201350 13-18. (doi:10.1016/j.peptides.2013.09.011)

13 Craig CL, Marshall AL, Sjostrom M, Bauman AE, Booth ML, Ainsworth BE, Pratt M, Ekelund U, Yngve A, Sallis JF \& Oja P. International physical activity questionnaire: 12 -country reliability and validity. Medical Science and Sports Exercise 200335 1381-1395.

14 Bruce RA, Kusumi F \& Hosmer D. Maximal oxygen intake and nomographic assessment of functional aerobic impairment in cardiovascular disease. American Heart Journal 197385 546-562.

15 Institute for Medical Informatics Statistics and Documentation: Wed-Based Patient Randomization Service for Multi-Center Clinical Trials. Medical University of Graz. Retrieved from www.randomizer.at. Accessed June 2, 2012.

16 Daskalopoulou SS, Khan NA, Quinn RR, Ruzicka M, McKay DW, Hackam DG, Rabkin SW, Rabi DM, Gilbert RE, Padwal RS et al. The 2012 Canadian hypertension education program recommendations for the 
management of hypertension: blood pressure measurement, diagnosis, assessment of risk, and therapy. Canadian Journal of Cardiology $2012 \mathbf{2 8}$ 270-287. (doi:10.1016/j.cjca.2012.02.018)

17 Harris JA \& Benedict FG. A biometric study of human basal metabolism. PNAS 19184 370-373. (doi:10.1073/pnas.4.12.370)

18 Swain DP, Brawner CA, American College of Sports Medicine \& American College of Sports Medicine. ACSM's resource manual for Guidelines for exercise testing and prescription. p 151. Philadelphia: Wolters Kluwer Health/Lippincott Williams \& Wilkins, 2014.

19 Hill AB. The environment and disease: association or causation? Proceedings of the Royal Society of Medicine 196558 295-300.

20 Matsuo T, Saotome K, Seino S, Shimojo N, Matsushita A, Iemitsu M, Ohshima H, Tanaka K \& Mukai C. Effects of a low-volume aerobic-type interval exercise on $\mathrm{VO}_{2}$ max and cardiac mass. Medicine and Science in Sports and Exercise 201446 42-50. (doi:10.1249/MSS. Ob013e3182a38da8)

21 Murphy MH, Blair SN \& Murtagh EM. Accumulated versus continuous exercise for health benefit: a review of empirical studies. Sports Medicine 200939 29-43. (doi:10.2165/00007256-200939010-00003)

22 Tremblay MS, Warburton DE, Janssen I, Paterson DH, Latimer AE, Rhodes RE, Kho ME, Hicks A, Leblanc AG, Zehr L et al. New Canadian physical activity guidelines. Applied Physiology, Nutrition, and Metabolism 201136 36-46. (doi:10.1139/H11-009)

23 Stengel A, Hofmann T, Goebel-Stengel M, Elbelt U, Kobelt P \& Klapp BF. Circulating levels of irisin in patients with anorexia nervosa and different stages of obesity - correlation with body mass index. Peptides 201339 125-130. (doi:10.1016/j.peptides.2012.11.014)

24 Moreno-Navarrete JM, Ortega F, Serrano M, Guerra E, Pardo G, Tinahones F, Ricart W \& Fernandez-Real JM. Irisin is expressed and produced by human muscle and adipose tissue in association with obesity and insulin resistance. Journal of Clinical Endocrinology and Metabolism 201398 E769-E778. (doi:10.1210/jc.2012-2749)
25 Brown SP MW \& Eason JM. In Exercise Physiology: Basis of Human Movement in Health and Disease p 90. Baltimore, MD: Lippincott Williams \& Wilkins, 2005.

26 Hecksteden A, Wegmann M, Steffen A, Kraushaar J, Morsch A, Ruppenthal S, Kaestner L \& Meyer T. Irisin and exercise training in humans - results from a randomized controlled training trial. BMC Medicine 201311 235. (doi:10.1186/1741-7015-11-235)

27 Kraemer RR, Shockett P, Webb ND, Shah U \& Castracane VD. A transient elevated irisin blood concentration in response to prolonged, moderate aerobic exercise in young men and women. Hormone and Metabolic Research 201446 150-154. (doi:10.1055/s-0033-1355381)

28 Kurdiova T, Balaz M, Vician M, Maderova D, Vlcek M, Valkovic L, Srbecky M, Imrich R, Kyselovicova O, Belan V et al. Are skeletal muscle $\&$ adipose tissue Fndc5 gene expression and irisin release affected by obesity, diabetes and exercise? In vivo \& in vitro studies. Journal of Physiology 2014592 1091-1107.

29 Park KH, Zaichenko L, Brinkoetter M, Thakkar B, Sahin-Efe A, Joung KE, Tsoukas MA, Geladari EV, Huh JY, Dincer F et al. Circulating irisin in relation to insulin resistance and the metabolic syndrome. Journal of Clinical Endocrinology and Metabolism 201398 4899-4907. (doi:10.1210/ jc.2013-2373)

30 Erickson HP. Irisin and FNDC5 in retrospect: an exercise hormone or a transmembrane receptor? Adipocytes 20132 289-293. (doi:10.4161/ adip.26082)

31 Raschke S, Elsen M, Gassenhuber H, Sommerfeld M, Schwahn U, Brockmann B, Jung R, Wisloff U, Tjonna AE, Raastad T et al. Evidence against a beneficial effect of irisin in humans. PLOS ONE $2013 \mathbf{8}$ e73680. (doi:10.1371/journal.pone.0073680)

32 Bostrom PA, Fernandez-Real JM \& Mantzoros C. Irisin in humans: recent advances and questions for future research. Metabolism 201463 178-180. (doi:10.1016/j.metabol.2013.11.009)

Received 12 March 2014

Revised version received 22 May 2014

Accepted 11 June 2014 